\title{
Multiple Outflows in (Proto)-Planetary Nebulae
}

\author{
Cox \\ Institut d'Astrophysique Spatiale, F-91405 Orsay Cedex, France
}

\begin{abstract}
This paper describes the structural changes occuring in the circumstellar envelopes of stars evolving from the AGB to the planetary nebula phase, with an emphasis on the role of multiple outflows.
\end{abstract}

\section{Introduction}

During the rapid transition between the AGB and planetary nebula (PN) phase, the proto-planetary nebulae (PPNe), e.g., Kwok (1993), the role played by highvelocity winds which interact with the slowly expanding AGB envelope has been recognized as essential in the shaping of the PNe. However, the details of the interaction and the precise evolution from the symmetric AGB envelope to the asymmetries which characterize PNe are not well understood. The high-velocity winds induce irreversible structural changes in the AGB remnant molecular envelope and when the central star begins to emit ultraviolet radiation further destruction takes place via the effects of photo-ionisation and photo-evaporation.

\section{Proto-Planetary Nebulae}

AFGL 2688 (the 'Egg nebula') is one of the prime examples of a PPN which has evolved from the AGB phase about a hundred years ago. The nearly circular slowly expanding AGB envelope (with a diameter of about $20^{\prime \prime}$ ) is shocked by a warm, optically thin, fast wind. This interaction is seen in high spatial resolution observations in the line emission of $\mathrm{CO} \mathrm{J}=2 \rightarrow 1$ and $\mathrm{H}_{2} 1 \rightarrow 0 \mathrm{~S}(1)$ (Cox et al. 2000, 2001 and references therein). In the velocity integrated $\mathrm{CO}$ map, the distribution of the $\mathrm{CO}$ emission consists of a central core $\sim 4^{\prime \prime}$ in diameter, with extensions in both the north-south and east-west directions. The CO J=2 $\rightarrow 1$ and $\mathrm{H}_{2} 1 \rightarrow 0 \mathrm{~S}(1)$ kinematics provide definite evidence of two distinct highvelocity outflow directions in AFGL 2688, one along a north-south axis at a P.A. of $17^{\circ}$, and the other in a roughly orthogonal direction east-west. These two main outflows are resolved into a series of more collimated, bipolar outflows which are symmetric in direction and velocity about the center: four collimated outflows in the east-west direction, and three in the north-south direction. Their kinematical ages range from $\sim 100$ to $\sim 1000$ years. The observed $\mathrm{CO}$ gas is entrained by high-velocity jets which could be atomic or ionized. The correlation of the $\mathrm{CO}$ outflows with the $\mathrm{H}_{2} 1 \rightarrow 0 \mathrm{~S}(1)$ emission provides direct evidence for their interaction with the nearly spherical envelope ejected on the AGB (Cox et al. 2001; Kastner et al. 2001). 
The presence of multiple, collimated outflows in two, roughly orthogonal directions cannot be explained by the standard two-winds model (e.g., Balick 1987). In this model, a fast spherical wind interacts with the slowly expanding AGB envelope and develops a bipolar outflow due to the presence of an equatorial density enhancement, commonly assumed to be the result of a close binary companion affecting the mass expelled by the AGB star (Morris 1987). Other PPNe with morphologies similar to AFGL 2688 have been observed (e.g., Kwok et al. 1998) and examples of multiples outflows are documented in the case of CRL 618 (e.g., Trammer 2000). Whatever detailed processes are involved, the high-velocity winds must be intimately linked to the abrupt transition in the evolution of the star after the AGB phase. One important consequence of the jets is their shaping effect on the molecular envelope ejected on the AGB. They generate complex point symmetries in the envelope which later emerge in the ionized gas during the planetary nebula phase.

\section{Planetary Nebulae}

The interaction of multiple outflows and the onset of photo-ionization through the post-AGB phase alter completely the molecular AGB envelope. In spite of these destructive processes, a significant component of molecular gas is found in many PNe, even in highly evolved ones (e.g., Huggins et al. 1996). In most cases, the molecular gas is found around the waist of the ionized gas in toroidlike shapes and represent a main structural feature of the nebula. The molecular gas in $\mathrm{PNe}$ is characterized be a high degree of fragmentation, the molecular envelope consisting of many small $\left(\approx 10^{15} \mathrm{~cm}\right.$ in size $)$, dense $\left(10^{5} \mathrm{~cm}^{-3}\right)$ clumps slowly evaporating in the radiation of the central white dwarf (e.g., Huggins et al. 2002; O'Dell et al. 2002). The origin of these tiny structures remains open, but without these high densities, the globules would photo-evaporate and disperse in the strong radiation on timescales much shorter than the lifetime of the nebula. The inhomogeneities and the high densities in the AGB envelope are thus a prerequisite to the survival of the molecular gas throughout the evolution of PNe. Another aspect of the distribution of molecular gas is its global structure which preserves imprints of the early interaction of the envelope with collimated, bipolar outflows or jets just after the AGB phase.

A striking example of a PN containing key information on the physical processes that shaped the nebula is NGC 7027. The dense, inner envelope of this relatively young $(\approx 600 \mathrm{yr}) \mathrm{PN}$ has been ionized by the intense radiation field of the hot central star $\left(\mathrm{T} \sim 200,000 \mathrm{~K}\right.$ and $\left.\mathrm{L} \approx 7,700 \mathrm{~L}_{\odot}\right)$, but farther out the envelope is still molecular. Whereas the ionized gas has a morphology which is roughly consistent with a prolate ellipsoidal shell, the molecular hydrogen emission is found along a striking shell with fourfold symmetry which loops around the ionized gas (Fig. 1). The detailed structure is complex with multiple lobes and weak $\mathrm{H}_{2}$ emission linking the polar caps along the major axis. The four-lobed clover pattern shows holes in the brightness distribution, especially to the north-west and south-east (Fig. 1) where weak $\mathrm{H}_{2}$ emission is extending outwards from the main structure (Latter et al. 2000; Cox et al. 2002). Despite the complexity, there is a remarkable symmetry in the distribution with respect to the central star. Whereas the overall structure of NGC 7027 is roughly axi- 


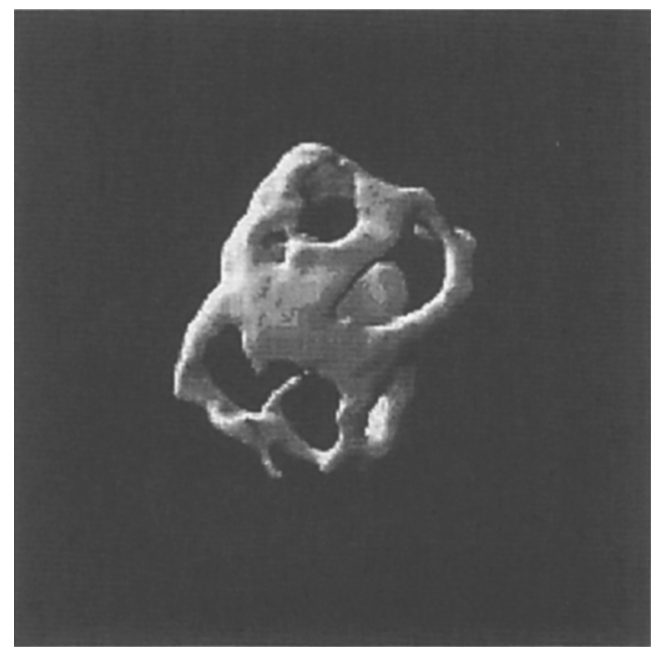

Figure 1. Three-dimensional representation of the $\mathrm{H}_{2} \quad 1 \rightarrow 0 \mathrm{~S}(1)$ in NGC 7027. The view shows surface contours of the $\mathrm{H}_{2}$ emission as seen from the front, i.e. as observed - from Cox et al. (2002).

symmetric, the remarkable series of holes or cavities in the molecular envelope are point symmetric about the central star both in position and velocity (Cox et al. 2002). These point symmetric holes are best understood as the result of recent multiple, bipolar outflows from the central star which pierced the molecular envelope of NGC 7027 during its evolution from the AGB to the present planetary nebula phase.

\section{Multiple Outflows and Envelope Shaping}

In summary, the above examples underscore the importance of collimated outflows in the shaping of planetary nebulae. These outflows, which occur at an early period when the star leaves the AGB, pierce and disrupt the circumstellar envelope. Recent mapping studies in molecular gas tracers have provided further examples of young PNe displaying unambiguous signs of interaction with high-velocity outflows. These studies include: KjPn 8 where powerful optical jets have excavated biconical cavities in the surrounding molecular envelope of this young PN (Forveille et al. 1998); BD $+30^{\circ} 3639$ where symmetric, highvelocity $\left(50 \mathrm{~km} \mathrm{~s}^{-1}\right)$ molecular bullets have been detected (Bachiller et al. 2000) which impact with the surrounding neutral gas; M1-16 where the outflows have digged large bipolar cavities along the bipolar axes and created symmetric lobes of molecular gas by entrainment (Huggins et al. 2000).

The complex, bipolar or multi-polar structures seen in the molecular envelopes of $\mathrm{PNe}$ are seen in the ionized gas of essentially all young PNe (Lopez 2000 and references therein; Sahai \& Trauger 1998). The phenomenon of wandering or precessing jets appears thus to be a basic mechanism in the stellar evolution of stars of a few solar masses at the end of the AGB phase. If a 
massive cool envelope is present at this stage, it will preserve the effects of the impacts of the outflows, due to the low sound speed of the cool gas, during the entire nebular lifetime (as in the case of the Helix - see Young et al. 1999). For less massive progenitors, the shaping will occur in an envelope which is already largely ionized and the structures will tend to dissipate much more rapidly due to the higher sound speed in this warm gas.

\section{Acknowledgements}

It is a pleasure to acknowledge collaborative work with R. Bachiller, T. Forveille, P.J. Huggins, R. Lucas, and J.-P. Maillard which form the basis of this contributed paper.

\section{References}

Bachiller, R., Forveille, T., Huggins, P.J., Cox, P., \& Maillard, J.-P. 2000, A\&A, 353, L5

Balick, B. 1987, AJ, 94, 671

Cox, P., Lucas, R., Huggins, P.J. et al. 2000, A\&A, 353, L25

Cox, P., Lucas, R., Huggins, P.J., \& Maillard, J.-P. 2001, in Post-AGB Objects as a Phase of Stellar Evolution, ed. R. Szczerba \& S.K. Górny, Astroph. Space Science (Kluwer) 265, 387

Cox, P., Huggins, P.J., Maillard, J.-P., et al. 2002, A\&A, 383, 603

Forveille, T., Huggins, P.J., Bachiller, R., \& Cox P. 1998, ApJ, 495, L111

Huggins, P.J., Bachiller, R., Cox, P., \& Forveille, T. 1996, A\&A, 315, 284

Huggins, P.J., Forveille, T., Bachiller, R., \& Cox, P. 2000, ApJ, 544, 889

Huggins, P.J., Forveille, T., Bachiller, R., et al. 2002, ApJ, 573, L55

Kastner, J.H, Weintraub, D.A., Gatley, I., \& Henn, L.A. 2001, ApJ, 546, 287

Kwok, S. 1993, ARAA, 31, 63

Kwok, S., Su, Y.L.S., \& Hrivnak, B.J. 1998, ApJ, 501, L117

Latter, W.B., Dayal, A., Bieging J.H., et al. 2000, ApJ, 539, 783

Lopez, J.A. 2000, Astrophysical Plasmas: Codes, Models, and Observations ed. J. Arthur, N. Brickhouse, \& J. Franco, Revista Mexicana de Astronomía y Astrofísica (Serie de Conferencias), 9, 201

Morris, M. 1987, PASP, 99, 1115

O'Dell, C.R., Balick, B., Hajian, A.R. et al. 2002, AJ, 123, 3329

Sahai, R., \& Trauger, J.T. 1998, AJ, 116, 1357

Trammell, S.R. 2000, in ASP Conference Series, Vol. 199, Asymmetrical Planetary nebulae II: From Origins to Microstructures, ed. J.H. Kastner, N. Soker \& S. Rappaport, 147

Young, K., Cox, P., Huggins, P.J., Forveille, T. \& Bachiller, R. 1999 ApJ, 522, 387 